\title{
Luminal-apposing stents for benign intraluminal strictures: a large United States multicenter study of clinical outcomes
}

\author{
Meir Mizrahi ${ }^{a^{*}}$, Yazan Fahmawi ${ }^{b^{*}}$, Lindsey Merritt ${ }^{a}$, Manoj Kumara, Benjamin Tharianc, Salman Ali Khanc, \\ Sumant Inamdarc, Neil Sharma ${ }^{d}$, Dushant Uppale, Vanessa M. Shamie, Mahmood Syed Kashiff, \\ Moamen Gabr', Douglas Pleskowh ${ }^{\mathrm{h}}$, Tyler M. Berzinh, Ted W. James', Michael Croglioi, \\ Todd H. Baron' , Douglas G. Adlerj
}

University of South Alabama, Mobile, AL; University of Arkansas for Medical Sciences, Little Rock, AR; Parkview Health, Fort Wayne, IN; University of Virginia Health System, Charlottesville, VA; Orange Regional Medical Center, Middletown, NY; University of Kentucky College of Medicine, Lexington, KY; Beth Israel Deaconess Medical Center, Harvard Medical School, Boston, MA; University of North Carolina at Chapel Hill; University of Utah School of Medicine, Salt Lake City, UT, USA

\section{Abstract}

Background The use of fully covered lumen-apposing metal stents (LAMS) for benign short gastrointestinal (GI) strictures has been reported. This study aimed to evaluate the safety and efficacy of LAMS for refractory GI strictures.

Methods A retrospective analysis was performed of patients who underwent LAMS placement for benign GI strictures in 8 United States centers. The primary outcomes were technical success and initial clinical response. Secondary outcomes were reintervention rate and adverse events.

Results A total of 51 patients underwent 61 LAMS placement procedures; 33 (64.7\%) had failed previous treatments. The most common stricture location was the pylorus ( $\mathrm{n}=17$ patients). Various sizes of stents were used, with 15-mm LAMS placed in 45 procedures, 20-mm LAMS in 14 procedures, and 10-mm LAMS in 2 procedures. The overall technical success, short-term clinical response and reintervention rate after stent removal were $100 \%, 91.8 \%$ and $31.1 \%$, respectively. Adverse events were reported in 17 (27.9\%) procedures, with stent migration being the most common (13.1\%). In subgroup analysis, both $15 \mathrm{~mm}$ and $20 \mathrm{~mm}$ stents had comparable short-term clinical response and adverse event rates. However, stent migration (15.6\%) was the most common adverse event with 15-mm LAMS while pain (14.3\%) was the most common with 20-mm LAMS. The reintervention rate was $80 \%$ at 200 -day follow up after stent removal.

Conclusions Using LAMS for treatment of short benign GI strictures is safe and effective. Larger LAMS, such as the new $20 \mathrm{~mm}$ in diameter, may have a lower stent migration rate compared to smaller diameter LAMS.

Keywords Lumen-apposing metal stent, gastrointestinal stricture, stent migration

Ann Gastroenterol 2020; 33 (6): 1-6
Conflict of Interest: Meir Mizrahi: Boston Scientific - Consultant. Yazan Fahmawi indicated no relevant financial relationships. Manoj Kumar indicated no relevant financial relationships. Benjamin Tharian: Boston Scientific - Consultant. Salman Ali Khan indicated no relevant financial relationships. Sumant Inamdar indicated no relevant financial relationships. Neil Sharma: Boston Scientific - Consultant. Dushant Uppal indicated no relevant financial relationships. Vanessa Shami indicated no relevant financial relationships. Mahmood Syed Kashif indicated no relevant financial relationships. Moamen Gabr indicated no relevant financial relationships. Douglas Pleskow: Boston scientific - Consultant. Tyler Berzin: Boston Scientific - Consultant. Douglas Adler: Boston Scientific - Consultant

*The first 2 authors contributed equally to this work

Correspondence to: Douglas G. Adler, MD, FACG, AGAF, FASGE, Professor of Medicine, Director, GI Fellowship Program,

Gastroenterology and Hepatology, University of Utah School of Medicine, Huntsman Cancer Center, 30N 1900E 4R118, Salt Lake City, Utah 84132, USA, e-mail: Douglas.adler@hsc.utah.edu

Received 10 April 2020; accepted 16 June 2020; published online 2 October 2020

DOI: https://doi.org/10.20524/aog.2020.0538
Department of a Gastroenterology and Hepatology, Advanced Endoscopy Unit, University of South Alabama, Mobile, AL (Meir Mizrahi, Lindsey Merritt, Manoj Kumar); ' Internal Medicine, University of South Alabama, Mobile, AL (Yazan Fahmawi); ${ }^{\mathrm{c}}$ Gastroenterology and Hepatology, University of Arkansas for Medical Sciences, Little Rock, AR (Benjamin Tharian, Salman Ali Khan, Sumant Inamdar); ${ }^{\mathrm{d} G a s t r o e n t e r o l o g y}$ and Hepatology, Parkview Health, Fort Wayne, IN (Neil Sharma); ${ }^{\mathrm{e}}$ Gastroenterology and Hepatology, University of Virginia Health System, Charlottesville, VA (Dushant Uppal, Vanessa M. Shami); ${ }^{\mathrm{f} G a s t r o e n t e r o l o g y}$ and Hepatology, Orange Regional Medical Center, Middletown, NY (Mahmood Syed Kashif); ${ }^{\mathrm{g}}$ Gastroenterology and Hepatology, University of Kentucky College of Medicine, Lexington, KY (Moamen Gabr); ${ }^{\text {h }}$ Gastroenterology and Hepatology, Beth Israel Deaconess Medical Center, Harvard Medical School, Boston, MA (Douglas Pleskow, Tyler M. Berzin); ${ }^{\mathrm{i}}$ Gastroenterology and Hepatology, University of North Carolina at Chapel Hill, Chapel Hill, North Carolina (Ted W. James, Michael Croglio, Todd H Baron); 'Gastroenterology and Hepatology, University of Utah School of Medicine, Salt Lake City, UT (Douglas G. Adler), USA 


\section{Introduction}

Benign gastrointestinal (GI) strictures can arise anywhere in the luminal GI tract as a result of a variety of causes, including inflammation, postoperative anastomosis, ulcer disease, and caustic ingestion [1]. Endoscopic therapy remains the mainstay management for the majority of these strictures, with several available modalities, including endoscopic dilatation, steroid injection, and fully covered self-expandable metal stent (FCSEMS) [2-4]. Although these modalities are relatively safe and effective, they are not without limitations. Endoscopic dilatation often requires multiple sessions, has a high rate of clinical recurrence, even with the addition of steroid injection, and entails a risk of perforation and bleeding [5]. FCSEMS are one option to overcome such limitations; however, these devices have a high rate of migration when placed in benign strictures [6]. This risk is the highest within the first month of stent placement, even if the stent is fixed with sutures or clips [6-8].

Lumen-apposing metal stents (LAMS) (Axios stent, Boston Scientific, Natick, Mass.) are fully covered stents with a $10-\mathrm{mm}$ length and diameters of 10, 15 and $20 \mathrm{~mm}$ (in the USA), with a wide flange at each end. These stents were initially developed for drainage of pancreatic fluid collections; however, they have been increasingly used in other settings, such as endoscopic ultrasound-directed transgastric endoscopic retrograde cholangiopancreatography, gallbladder drainage, and to treat short-segment benign GI strictures [9-12]. The biflanged design along with the short saddle length make the risk of stent migration relatively low [13]. Therefore, LAMS have emerged as a novel modality for treating benign GI strictures. In this study, we report the feasibility, safety and efficacy of treating benign GI strictures with LAMS in different centers across the United States.

\section{Patients and methods}

\section{Study population and data collection}

This study was a retrospective, single-arm, multicenter study that included patients $>18$ years old with benign GI strictures at 8 large tertiary referral centers in the United States. The demographic data, location of stricture, duration of stricture before LAMS placement, previous endoscopic treatment for the strictures, LAMS lengths, adverse events, rate of reintervention, procedural information, and follow-up period for each patient were extracted. Informed procedural consent was obtained from all patients. The study was approved by the institutional review board for human research at each participating institution. Patients were excluded from the study if they were less than 18 years old or the LAMS was placed for malignant strictures.

\section{Endoscopic technique}

The LAMS are commercially available in 3 different luminal diameters; $10 \mathrm{~mm}, 15 \mathrm{~mm}$, and $20 \mathrm{~mm}$. All stents have a saddle length of $10 \mathrm{~mm}$. The flange diameter increases in size with wider stents (21 mm, $24 \mathrm{~mm}$ and $29 \mathrm{~mm}$ ). A forward-viewing therapeutic endoscope or an oblique-viewing therapeutic linear echoendoscope was used for stent deployment in all cases. If felt to be warranted, a guidewire was introduced across the stricture under fluoroscopic guidance. The stent was then positioned and deployed across the stricture under endoscopic guidance. Depending on the endoscopist's discretion, the LAMS was removed using rat-tooth, biopsy forceps, and/or snare.

\section{Definitions}

Technical success was defined as successful LAMS deployment across the stricture, verified on endoscopic and/ or fluoroscopic views. Short-term clinical success was defined as resolution of symptoms with the indwelling LAMS in place. Long-term clinical success was defined as symptom resolution following LAMS removal and without reintervention. The last follow up was defined as the patient's last visit, whether in the clinic or via telecommunication.

\section{Study outcomes}

The primary outcomes in this study were to evaluate the overall technical success and short-term clinical success of LAMS placement for management of benign GI strictures and to compare the outcomes of the most widely used LAMS sizes, $15 \mathrm{~mm}$ and $20 \mathrm{~mm}$. Secondary outcomes were stent migration, reintervention rate due to recurrence of symptoms, and adverse events.

\section{Data analysis}

A Kaplan-Meier analysis was performed to determine the need for reintervention due to recurrence of symptoms after LAMS removal. The Kaplan-Meier analysis starting time was the LAMS extraction, while the endpoint was the last follow up. To compare the outcomes of the 2 sizes ( $15 \mathrm{~mm}$ vs. $20 \mathrm{~mm}$ ) of LAMS stents chi-squared analysis and/or Fischer's exact test were used for categorical variables, while a $t$-test was used to compare means of continuous variables. Frequencies and percentages were calculated using basic descriptive statistics.

\section{Results}

\section{Patient characteristics}

Fifty-one patients (20 male) with a mean age of 60.3 years underwent LAMS placement for 61 benign luminal strictures (Table 1): $10 \mathrm{~mm} \times 10 \mathrm{~mm}$ LAMS were placed in $2(3.2 \%)$ patients, $15 \mathrm{~mm} \times 10 \mathrm{~mm}$ LAMS in $36(70.6 \%)$, and $20 \mathrm{~mm}$ $\times 10 \mathrm{~mm}$ LAMS in $13(25.5 \%)$ patients. The mean stricture length was $8.3 \mathrm{~mm}$. Most strictures were located in the pylorus 
Table 1 Baseline characteristics and previous treatments

\begin{tabular}{|c|c|c|c|c|}
\hline Characteristics & $\begin{array}{l}\text { All patients } \\
\mathrm{n}=51\end{array}$ & $\begin{array}{c}15 \mathrm{~mm} \times 10 \mathrm{~mm} \\
\text { LAMS } \mathrm{n}=36\end{array}$ & $\begin{array}{l}20 \mathrm{~mm} \times 10 \mathrm{~mm} \\
\text { LAMS } \mathrm{n}=13\end{array}$ & P-value \\
\hline Age, mean, years & 60.3 & 58.6 & 63 & 0.88 \\
\hline Men, n (\%) & $20(39.2 \%)$ & $12(33.3 \%)$ & $6(46.2 \%)$ & 0.54 \\
\hline Weight, kg & 78.1 & 77.2 & 81.4 & 0.91 \\
\hline Number of strictures & 61 & 45 & 14 & \\
\hline Stricture length, mean (SD), mm & $8.3(2.3)$ & $7.4(2.3)$ & $10(2.3)$ & 0.53 \\
\hline Duration of the stricture prior to LAMS, mean (SD), months & $17.3(20.9)$ & $17.5(20.9)$ & $19.8(21.1)$ & 0.9421 \\
\hline $\begin{array}{l}\text { Location of stricture } \\
\text { Esophagogastric anastomoses } \\
\text { gastro-Jejunal anastomosis } \\
\text { Pylorus } \\
\text { Ileo-colonic } \\
\text { Duodenum } \\
\text { Esophagus } \\
\text { Gastric body } \\
\text { Sigmoid } \\
\text { Other }\end{array}$ & $\begin{array}{c}10(19.6 \%) \\
11(21.6 \%) \\
17(33.3 \%) \\
2(3.9 \%) \\
4(7.8 \%) \\
2(3.9 \%) \\
1(2 \%) \\
1(2 \%) \\
3(5.9 \%)\end{array}$ & $\begin{array}{c}5(13.9 \%) \\
9(25 \%) \\
13(36.1 \%) \\
2(5.6 \%) \\
4(11.1 \%) \\
1(2.8 \%) \\
1(2.8 \%) \\
1(2.8 \%) \\
0(0 \%)\end{array}$ & $\begin{array}{c}4(30.8 \%) \\
2(15.4 \%) \\
4(30.8 \%) \\
0 \\
0 \\
0 \\
0 \\
0 \\
3(23 \%)\end{array}$ & $\begin{array}{c}0.48 \\
>0.99 \\
>0.99 \\
>0.99 \\
>0.99 \\
>0.99 \\
>0.99 \\
>0.99 \\
0.015\end{array}$ \\
\hline $\begin{array}{l}\text { Previous treatment } \\
\text { Previous balloon dilation, } \mathrm{n}(\%) \\
\text { Previous balloon dilation plus steroid injection, n (\%) } \\
\text { Previous needle-knife, } \mathrm{n}(\%) \\
\text { Previous traditional FCSEMS, n (\%) }\end{array}$ & $\begin{array}{l}33(64.7 \%) \\
27(52.9 \%) \\
14(27.4 \%) \\
2(3.9 \%) \\
6(11.8 \%)\end{array}$ & $\begin{array}{l}22(61.1 \%) \\
17(47.2 \%) \\
8(15.7 \%) \\
2(5.6 \%) \\
4(7.8 \%)\end{array}$ & $\begin{array}{l}10(76.9 \%) \\
8(57.1 \%) \\
5(38.5 \%) \\
0 \\
2(15.4 \%)\end{array}$ & $\begin{array}{c}0.50 \\
0.53 \\
0.29 \\
>0.99 \\
0.65\end{array}$ \\
\hline
\end{tabular}

LAMS, lumen-apposing metal stent; FCSEMS, fully covered self-expandable metal stent; SD, standard deviation

(33.3\%), followed by gastrojejunal anastomoses (21.6\%), esophagogastric anastomoses (19.6\%), duodenum (7.8\%), other (5.9\%), ileocolonic (3.9\%), esophagus (3.9\%), gastric body (2\%), and sigmoid colon (2\%). Eighteen (35.3\%) patients had had no previous stricture treatments, 27 (52.9\%) had previously undergone endoscopic dilation alone, 14 (27.5\%) had previously undergone endoscopic balloon dilation combined with steroid injection, 2 (3.9\%) had undergone prior needle-knife strictureplasty, and 6 (11.8\%) had received previous FCSEMS. Clinical symptoms had been present for a mean of 17.3 months.

\section{Procedural and clinical outcomes}

Table 2 shows the procedure and clinical outcomes. LAMS were deployed across strictures successfully in all procedures; the technical success rate was $100 \%$, regardless of the stent size. Short-term clinical success was achieved in 56/61 (91.8\%) procedures. The median procedure time was $17.5 \mathrm{~min}$ (range 5-38). Among the 61 LAMS placed, 44 (72\%) were either removed endoscopically or passed spontaneously, and 13 (21.3\%) were still in place during the follow-up period. The fate of the remaining 4 stents was unknown, as patients either died or went to a hospice during the follow-up period with the stent in situ. Mean stent indwell time was 71.7 days. Long-term clinical success was achieved in 18/61 (29.5\%).

Adverse events were reported in 17 (27.9\%) patients after LAMS placement. Stent migration was the most common adverse event $(13.1 \%)$, followed by pain $(6.6 \%)$, occlusion
(4.9\%), bleeding (1.6\%), and the formation of a proximal or distal stricture (1.6\%). Reintervention after stent removal was performed in $19(31.1 \%)$ cases. The reintervention rate after stent removal was almost $80 \%$ at 200 days of follow up (Fig. 1). Balloon dilation after stent removal and surgical intervention were performed in $9(14.7 \%)$ and $5(8.2 \%)$ cases, respectively. A new LAMS was placed (similar size or different size) because of recurrence of symptoms in $11(18 \%)$ cases. The mean time to reintervention was 73.3 days. All patients had at least one follow-up visit and the mean follow-up period was 173.8 days.

\section{5- vs. 20-mm LAMS}

Tables 1 and 2 show the basic characteristics and procedural outcomes based on LAMS size. No statistical differences between the 2 groups were noted in the baseline characteristics. Twenty-two (61.1\%) patients who received 15-mm LAMS had undergone at least one previous intervention, while 10 patients (76.9.1\%) with 20-mm LAMS had undergone at least one previous intervention. The most common prior intervention in both groups was balloon dilation, at $47.2 \%$ and $57.1 \%$ for the 15- and 20-mm LAMS groups, respectively. However, these differences were not statistically significant.

Regarding the procedural and clinical outcomes, the $15-\mathrm{mm}$ LAMS and the 20-mm LAMS had comparable short-term clinical success, $91.1 \%$ and $92.9 \%$, respectively. Both sizes of stent had essentially the same adverse event rate: $28.9 \%$ for the 15-mm LAMS and $28.6 \%$ for the 20 -mm LAMS. However, the 
Table 2 Procedure and outcome variables

\begin{tabular}{|c|c|c|c|c|}
\hline Etiology & All procedures & $15 \mathrm{~mm}(\mathrm{n}=45)$ & $20 \mathrm{~mm}(\mathrm{n}=14)$ & P-value \\
\hline Procedure time, median (range), min & $17.5(5-138)$ & $21(5-138)$ & $21(8-78)$ & 0.83 \\
\hline Technical success, n (\%) & $61(100 \%)$ & $45(100 \%)$ & $14(100 \%)$ & $>0.99$ \\
\hline Short-term clinical success, n (\%) & $56(91.8 \%)$ & $41(91.1 \%)$ & $13(92.9 \%)$ & $>0.99$ \\
\hline Stent indwell time, mean (SD), days & $71.7(106.7)$ & $72.0(104.2)$ & $71.2(106.7)$ & $>0.99$ \\
\hline Stent removed & $36(59 \%)$ & $28(62.2 \%)$ & $6(42.9 \%)$ & 0.23 \\
\hline $\begin{array}{l}\text { Adverse events } \\
\text { Stent migration } \\
\text { Pain } \\
\text { Bleeding } \\
\text { Perforation } \\
\text { Occlusion } \\
\text { Adjacent stricture }\end{array}$ & $\begin{array}{c}17(27.9 \%) \\
8(13.1 \%) \\
4(6.6 \%) \\
1(1.6 \%) \\
0(0 \%) \\
3(4.9 \%) \\
1(1.6 \%)\end{array}$ & $\begin{array}{c}13(28.9 \%) \\
7(15.6 \%) \\
2(4.4 \%) \\
1(2.2 \%) \\
0(0 \%) \\
2(4.4 \%) \\
1(2.2 \%)\end{array}$ & $\begin{array}{c}4(28.6 \%) \\
1(7.1 \%) \\
2(14.3 \%) \\
0(0 \%) \\
0(0 \%) \\
1(7.1 \%) \\
0\end{array}$ & $\begin{array}{c}>0.99 \\
0.67 \\
0.24 \\
>0.99 \\
>0.99 \\
0.56 \\
>0.99\end{array}$ \\
\hline $\begin{array}{l}\text { Re-intervention after stent removal } \\
\text { Balloon dilation } \\
\text { Surgical resection } \\
\text { Stent replacement }\end{array}$ & $\begin{array}{l}19(31.1 \%) \\
9(14.7 \%) \\
5(8.2 \%) \\
11(18 \%)\end{array}$ & $\begin{array}{c}13(28.9 \%) \\
6(13.3 \%) \\
4(8.9 \%) \\
8(17.8 \%)\end{array}$ & $\begin{array}{c}4(28.6 \%) \\
3(21.4 \%) \\
0(0 \%) \\
1(7.1 \%)\end{array}$ & $\begin{array}{l}0.7 \\
0.4 \\
0.25 \\
0.33\end{array}$ \\
\hline Time for reintervention, mean (SD), weeks & $23.8(23.5)$ & $24.7(23.5)$ & $34(24.2)$ & 0.54 \\
\hline Follow up, mean (SD), days & $173.8(260.1)$ & $179.3(263.5)$ & $107.7(262.2)$ & 0.38 \\
\hline
\end{tabular}

SD, standard deviation

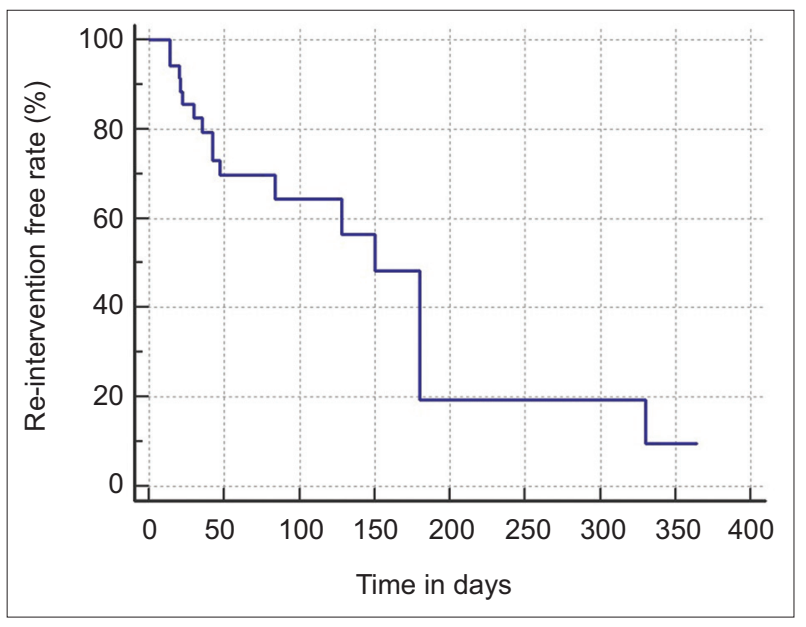

Figure 1 Rate of reintervention after lumen-apposing metal stent removal. In this figure, the starting point is the date of stent removal, while the endpoint is the last follow up

most common complication in the 15-mm LAMS group was stent migration which occurred in 7 patients (15.6\%), whereas the most common complication in the 20-mm LAMS group was postprocedural pain, reported in 2 cases (14.3\%). Other reported complications for the 15-mm LAMS group were postprocedural pain $(4.4 \%)$, occlusion $(4.4 \%)$, bleeding $(2.2 \%)$, and adjacent stricture (2.2\%). For the 20-mm LAMS group, one case of stent migration (7.1\%) and one case of stent occlusion (7.1\%) were reported. No statistically significant differences were found between the adverse events in the 2 groups (Table 2).

The reintervention rate after stent removal was comparable in the 20-mm LAMS and the 15-mm LAMS groups: $28.6 \%$ and $28.9 \%$, respectively $(\mathrm{P}=0.7)$. The rate of reintervention with balloon dilation after stent removal was higher (21.4\%) in the 20-mm LAMS compared to the 15-mm LAMS group (13.3\%), but the difference was not statistically significant $(\mathrm{P}=0.4)$. Surgical resection was performed in 5 cases $(8.2 \%)$ of the 15 mm LAMS group, whereas none was performed in the $20-\mathrm{mm}$ group. One stent $(7.1 \%)$ was replaced with another LAMS in the 20 -mm LAMS, whereas 8 stents (17.8\%) were replaced with another LAMS in the 15-mm LAMS group, with no statistically significant difference between the 2 groups $(\mathrm{P}=0.33)$.

\section{Discussion}

In this multicenter study, we demonstrated that LAMS are feasible, effective and safe for the endoscopic management of benign GI strictures. Given their unique design and shape, LAMS are widely used for a variety of clinical indications beyond pancreatic fluid drainage. However, data are emerging about the use of LAMS in the treatment of benign GI strictures. Benign GI strictures can be difficult to manage by conventional endoscopic methods, such as balloon dilatation, steroid injection or by stenting using FCSEMS. In the present study, $61 \%$ of the patients had failed previous treatment.

In this study, technical success of LAMS placement was achieved in all patients (100\%), regardless of the stricture etiology, the site or the size of stent. The median procedure time was $17.5 \mathrm{~min}$ (range 5-138) and the procedure was welltolerated by all patients. Previous studies have also reported similar feasibility of LAMS placement $[9,14,15]$. The shortterm clinical response was $91.8 \%$, regardless of stent size, comparable to the short-term clinical response reported in other studies (90\% and 90.5\%) $[9,13]$. However, it is slightly 
lower than the short-term clinical response reported by Bazerbachi et al (96.4\%) [14]. This small difference might be due to the stricture characteristics and/or stent sizes used in the 2 studies. To our knowledge, this study is the first to compare 2 LAMS sizes, $15 \mathrm{~mm}$ and $20 \mathrm{~mm}$. The short-term clinical success for the 15-mm LAMS group was comparable to that of the 20 -mm LAMS ( $91.1 \%$ vs. $92.9 \%$, respectively).

Overall, adverse events were reported in 17 procedures (27.9\%), comparable to the overall adverse events reported in a meta-analysis of 8 studies (29.9\%) [16]. Although the 2 stent sizes had the same rate of adverse events (28.9\% for 15 mm LAMS and $28.6 \%$ for 20-mm LAMS), stent migration was the most common adverse event in the 15-mm LAMS group (15.6\%) whereas postprocedural pain was the most common adverse event in the 20-mm LAMS group (14.3\%).

Stent migration has been a well-recognized limitation of FCSEMS for the management of benign GI stricture, with a rate of $31.1 \%$ [2]. In our study, the overall stent migration rate was $13.1 \%$. This lower migration rate of LAMS as compared to FCSEMS may be attributed to the LAMS stent design with the 2 flanges that distribute pressure across the stricture evenly, providing anchorage and reducing the risk of stent migration [13]. This effect was more pronounced with 20-mm LAMS: there was only one case of stent migration in this group, suggesting that the use of larger diameter LAMS may prolong the stent indwell time and may potentially lead to an increase in therapeutic yield.

Although LAMS has a good short-term clinical response, the reintervention rate is still high, as was demonstrated in this study, in which $80 \%$ of cases required reintervention at 200 days of follow up. Bazerbachi et al also reported a high reintervention rate, with $75 \%$ of cases requiring reintervention at 300 days of follow up [14]. In the current study, both stent sizes had the same reintervention rate after being removed. Although the difference was not statistically significant, surgical reintervention was required only in one case in the 20-mm LAMS group compared to 6 cases in the 15-mm LAMS group. The high reintervention rate raises concern about the cost-effectiveness of LAMS in the management of benign GI strictures, given the fact that LAMS placement is an expensive procedure. In a study evaluating the economical efficacy of LAMS in the management of benign GI strictures, Hallac et al found that LAMS placement may have an economic advantage over recurrent dilation after the third dilation [17]. In the present study, most of the strictures had already failed previous treatments. Therefore, given its tolerability and safety, LAMS might be considered as a destination therapy, especially in poor surgical candidates.

This study had several limitations that included its retrospective design, variations in the stricture characteristics and causes, different procedure techniques, as well as the lack of a control arm.

In conclusion, this study demonstrated that LAMS are feasible and safe, and have good short-term effectiveness for use in short, benign luminal GI strictures. Both LAMS sizes (15 $\mathrm{mm}$ and $20 \mathrm{~mm}$ ) have comparable efficacy and safety profiles. However, the large diameter stent $(20 \mathrm{~mm})$ has a lower stent migration rate compared to the small diameter stent $(15 \mathrm{~mm})$. Therefore, larger prospective trials comparing different stent types and sizes are warranted.

\section{Summary Box}

\section{What is already known:}

- The management of benign gastrointestinal (GI) strictures has been challenging with the available endoscopic modalities (endoscopic balloon dilation, intralesional steroid injection, and fully covered self-expanding metal stents)

- Lumen-apposing metal stents (LAMS) were originally developed for endoscopic ultrasoundguided drainage of pancreatic fluid collections

- Given its unique design and shape, LAMS have emerged as a novel modality for management of refractory benign GI strictures

\section{What the new findings are:}

- Overall technical, short-term and long-term success rates were $100 \%, 91.8 \%$ and $24.6 \%$. The overall rate of adverse events was $27.9 \%$, with stent migration being the most common (13.1\%)

- LAMS of $15 \mathrm{~mm}$ and $20 \mathrm{~mm}$ in diameter had comparable short-term clinical success: $91.1 \%$ and $92.9 \%$, respectively; their adverse events rates were also the same: $28.6 \%$ for 15 -mm LAMS and $28.9 \%$ for 20-mm LAMS

- The most common complication in the $15-\mathrm{mm}$ LAMS group was stent migration (15.6\%), while postprocedural abdominal pain was the most common complication (14.3\%) in the $20-\mathrm{mm}$ LAMS group

- Both stent sizes had comparable reintervention rate after stent removal: $28.9 \%$ and $28.6 \%$, respectively

\section{References}

1. Kochhar R, Kochhar S. Endoscopic balloon dilation for benign gastric outlet obstruction in adults. World J Gastrointest Endosc 2010;2:29-35.

2. Fuccio L, Hassan C, Frazzoni L, Miglio R, Repici A. Clinical outcomes following stent placement in refractory benign esophageal stricture: a systematic review and meta-analysis. Endoscopy 2016;48:141-148.

3. Jain D, Sandhu N, Singhal S. Endoscopic electrocautery incision therapy for benign lower gastrointestinal tract anastomotic strictures. Ann Gastroenterol 2017;30:473-485.

4. Ramage JI Jr, Rumalla A, Baron $\mathrm{TH}$, et al. A prospective, randomized, double-blind, placebo-controlled trial of endoscopic steroid injection therapy for recalcitrant esophageal peptic strictures. Am J Gastroenterol 2005;100:2419-2425.

5. Pereira-Lima JC, Ramires RP, Zamin I Jr, Cassal AP, Marroni CA, Mattos AA. Endoscopic dilation of benign esophageal strictures: report on 1043 procedures. Am J Gastroenterol 1999;94:1497-1501.

6. Hirdes MM, Siersema PD, Vleggaar FP. A new fully covered metal stent for the treatment of benign and malignant dysphagia: a 
prospective follow-up study. Gastrointest Endosc 2012;75:712-718.

7. Irani S, Baron TH, Gluck M, Gan I, Ross AS, Kozarek RA. Preventing migration of fully covered esophageal stents with an over-the-scope clip device (with videos). Gastrointest Endosc 2014;79:844-851.

8. Fujii LL, Bonin EA, Baron TH, Gostout CJ, Wong Kee Song LM. Utility of an endoscopic suturing system for prevention of covered luminal stent migration in the upper GI tract. Gastrointest Endosc 2013;78:787-793.

9. Santos-Fernandez J, Paiji C, Shakhatreh M, et al. Lumen-apposing metal stents for benign gastrointestinal tract strictures: an international multicenter experience. World J Gastrointest Endosc 2017;9:571-578.

10. Walter D, Will U, Sanchez-Yague A, et al. A novel lumen-apposing metal stent for endoscopic ultrasound-guided drainage of pancreatic fluid collections: a prospective cohort study. Endoscopy 2015;47:63-67.

11. Kedia P, Sharaiha RZ, Kumta NA, Kahaleh M. Internal EUSdirected transgastric ERCP (EDGE): game over. Gastroenterology 2014;147:566-568.
12. Kalva NR, Vanar V, Forcione D, Bechtold ML, Puli SR. Efficacy and safety of lumen apposing self-expandable metal stents for EUS guided cholecystostomy: a meta-analysis and systematic review. Can J Gastroenterol Hepatol 2018;2018:7070961.

13. Yang D, Nieto JM, Siddiqui A, et al. Lumen-apposing covered selfexpandable metal stents for short benign gastrointestinal strictures: a multicenter study. Endoscopy 2017;49:327-333.

14. Bazerbachi F, Heffley JD, Abu Dayyeh BK, et al. Safety and efficacy of coaxial lumen-apposing metal stents in the management of refractory gastrointestinal luminal strictures: a multicenter study. Endosc Int Open 2017;5:E861-E867.

15. Irani S, Jalaj S, Ross A, Larsen M, Grimm IS, Baron TH. Use of a lumen-apposing metal stent to treat GI strictures (with videos). Gastrointest Endosc 2017;85:1285-1289.

16. Mohan BP, Chandan S, Garg R, et al. Lumen-apposing metal stents, fully covered self-expanding metal stents, and biodegradable stents in the management of benign of GI strictures: a systematic review and meta-analysis. J Clin Gastroenterol 2019;53:560-573.

17. Hallac A, Srikureja W, Liu E, Dhumal P, Thatte A, Puri N. Economical effect of lumen apposing metal stents for treating benign foregut strictures. World J Gastrointest Endosc 2018;10:294-300. 